\title{
ON THE AMPLITUDE EQUATION OF APPROXIMATE SURFACE WAVES ON THE PLASMA-VACUUM INTERFACE
}

\author{
PAOLO SECCHI
}

\begin{abstract}
In this paper we present a recent result about the propagation of weakly nonlinear surface waves on a plasma-vacuum interface. In the plasma region we consider the equations of incompressible magnetohydrodynamics, while in vacuum the magnetic and electric fields are governed by the Maxwell equations. A surface wave propagate along the plasma-vacuum interface, when it is linearly weakly stable.

Following the approach of Alì and Hunter, we measure the amplitude of the surface wave by the normalized displacement of the interface in a reference frame moving with the linearized phase velocity of the wave, and obtain that it satisfies an asymptotic nonlocal, Hamiltonian evolution equation with quadratic nonlinearity. We show the local-in-time existence of smooth solutions to the Cauchy problem for the amplitude equation in noncanonical variables, and we derive the regularity of the first order corrections of the asymptotic expansion.
\end{abstract}

\section{INTRODUCTION}

Plasma-vacuum interface problems appear in the mathematical modeling of plasma confinement by magnetic fields in thermonuclear energy production (as in Tokamaks and Stellarators; see, e.g., [4]). In this model, the plasma is confined inside a perfectly conducting rigid wall and isolated from it by a region containing very low density plasma, which may qualify as vacuum, due to the effect of strong magnetic fields. In Astrophysics, the plasma-vacuum interface problem can be used for modeling the motion of a star or the solar corona when magnetic fields are taken into account.

For the sake of simplicity, in [11] we consider the plasma-vacuum interface problem in two-dimensions, with the coupling of the incompressible MHD equations in the plasma region and the Maxwell equations in the vacuum region. The solution is close to a stationary basic state with parallel magnetic fields at the flat interface.

To study the time evolution of the plasma-vacuum interface we follow the approach of Alì and Hunter in [1] and we show that, in a unidirectional surface wave, the normalized displacement $x_{2}=\varphi\left(t, x_{1}\right)$ of a weakly stable surface wave along the interface, in a reference frame moving with the linearized phase velocity of the wave, satisfies the quadratically nonlinear, nonlocal asymptotic equation

$$
\varphi_{t}+\frac{1}{2} \mathbb{H}\left[\Phi^{2}\right]_{x x}+\Phi \varphi_{x x}=0, \quad \Phi=\mathbb{H}[\varphi] .
$$

Here $\mathbb{H}$ denotes the Hilbert transform defined by

$$
\mathbb{H}[\varphi](x)=\frac{1}{\pi} \text { p.v. } \int_{-\infty}^{+\infty} \frac{\varphi(y)}{x-y} d y,
$$

and such that

$$
\mathbb{H}\left[e^{i k x}\right]=-i \operatorname{sgn}(\mathrm{k}) \mathrm{e}^{\mathrm{ikx}}, \quad \mathcal{F}[\mathbb{H}[\varphi]]=-\mathrm{i} \operatorname{sgn}(\mathrm{k}) \mathcal{F}[\varphi],
$$

for $\mathcal{F}$ denoting the Fourier transformation.

Equation (1) coincides with the amplitude equation for nonlinear Rayleigh waves [5] and currentvortex sheets in incompressible MHD [1,2]. It is interesting that exactly the same equation appears for

Date: February 23, 2015.

2010 Mathematics Subject Classification. Primary: 76W05; Secondary: 35Q35, 76E17, 76E25, 35R35, 76B03.

Key words and phrases. Incompressible Magneto-Hydrodynamics, Maxwell equations, plasma-vacuum interface.

The author is supported by the national research project PRIN 2012 "Nonlinear Hyperbolic Partial Differential Equations, Dispersive and Transport Equations: theoretical and applicative aspects". 
the incompressible plasma-vacuum interface problem, where in the vacuum part the electric and magnetic fields are ruled by the Maxwell equations. The derivation of the same equation confirms that (1) is a canonical model equation for nonlinear surface wave solutions of hyperbolic conservation laws, analogous to the inviscid Burgers equation for bulk waves. Equation (1) also admits the alternative spatial form

$$
\varphi_{t}+[\mathbb{H}, \Phi] \Phi_{x x}+\mathbb{H}\left[\Phi_{x}^{2}\right]=0,
$$

where $[\mathbb{H}, \Phi]$ is the commutator of $\mathbb{H}$ with multiplication by $\Phi$, see [8]. The alternative form (2) shows that (1) is an equation of first order, due to a cancelation of the second order spatial derivatives appearing in (1).

By adapting the proof of [7], in [11] we show the local-in-time existence of smooth solutions to the Cauchy problem for amplitude equation in noncanonical variables, and we derive a blow up criterion. Numerical computations $[1,5]$ show that solutions of (1) form singularities in which the derivative $\varphi_{x}$ blows up, but $\varphi$ appears to remain continuous. As far as we know, the global existence of appropriate weak solutions is an open question.

In the present note, from the previous existence result for the solution to the amplitude equation we also derive the regularity of the first order asymptotic corrections of the plasma variables and magnetic and electric fields in vacuum.

\section{The Plasma-VACUUM INTERFACE PROBLEM}

We consider the equations of incompressible magneto-hydrodynamics (MHD), i.e. the equations governing the motion of a perfectly conducting inviscid incompressible plasma. In the case of a homogeneous plasma (the density $\rho \equiv$ const $>0$ ), the equations in a dimensionless form read:

$$
\left\{\begin{array}{l}
\partial_{t} \mathbf{v}+\nabla \cdot(\mathbf{v} \otimes \mathbf{v}-\mathbf{B} \otimes \mathbf{B})+\nabla q=0, \\
\partial_{t} \mathbf{B}-\nabla \times(\mathbf{v} \times \mathbf{B})=0, \\
\operatorname{div} \mathbf{v}=0, \operatorname{div} \mathbf{B}=0,
\end{array}\right.
$$

where $\mathbf{v}$ denotes the plasma velocity, $\mathbf{B}$ is the magnetic field (in Alfvén velocity units), $q=p+|\mathbf{B}|^{2} / 2$ is the total pressure, $p$ being the pressure.

For smooth solutions, system (3) can be written in equivalent form as a symmetric system

$$
\left\{\begin{array}{l}
\partial_{t} \mathbf{v}+(\mathbf{v} \cdot \nabla) \mathbf{v}-(\mathbf{B} \cdot \nabla) \mathbf{B}+\nabla q=0 \\
\partial_{t} \mathbf{B}+(\mathbf{v} \cdot \nabla) \mathbf{B}-(\mathbf{B} \cdot \nabla) \mathbf{v}=0 \\
\operatorname{div} \mathbf{v}=0
\end{array}\right.
$$

In addition the magnetic field must satisfy the constraint

$$
\operatorname{div} \mathbf{B}=0,
$$

which is preserved by the evolution in time if it is satisfied by the initial data.

Let $\Omega^{+}(t)$ and $\Omega^{-}(t)$ be space-time domains occupied by the plasma and the vacuum respectively, separated by an interface $\Gamma(t)$. That is, in the domain $\Omega^{+}(t)$ we consider system (4) governing the motion of the plasma and in the domain $\Omega^{-}(t)$ we have the Maxwell system

$$
\left\{\begin{array}{l}
\nu \partial_{t} \mathbf{H}+\nabla \times \mathbf{E}=0, \\
\nu \partial_{t} \mathbf{E}-\nabla \times \mathbf{H}=0,
\end{array}\right.
$$

describing the vacuum magnetic and electric fields $\mathbf{H}, \mathbf{E} \in \mathbb{R}^{3}$. Here, the equations are written in nondimensional form through a suitable scaling (see Mandrik-Trakhinin [9]), and $\nu=\frac{\bar{v}}{c}$, where $\bar{v}$ is the velocity of a uniform flow and $c$ is the speed of light in vacuum. If we choose $\bar{v}$ to be the speed of sound in vacuum, we have that $\nu$ is a small, even though fixed parameter. System (5) is supplemented by the divergence constraints

$$
\operatorname{div} \mathbf{H}=\operatorname{div} \mathbf{E}=0
$$

on the initial data. 
For the sake of simplicity we consider the case of two space dimensions and write

$$
\mathbf{v}=\left(v_{1}, v_{2}\right)^{T}, \quad \mathbf{B}=\left(B_{1}, B_{2}\right)^{T} .
$$

In the (three-dimensional) Maxwell equations (5) we assume that

$$
\mathbf{H}=\left(H_{1}, H_{2}, 0\right)^{T},
$$

and that there is no dependence of $\mathbf{H}$ on the third space variable $x_{3}$. It follows from (5) that $\mathbf{E}$ takes the form

$$
\mathbf{E}=(0,0, E)^{T},
$$

and the Maxwell equations reduce to

$$
\left\{\begin{array}{l}
\nu \partial_{t} H_{1}+\partial_{2} E=0, \\
\nu \partial_{t} H_{2}-\partial_{1} E=0, \\
\nu \partial_{t} E-\partial_{1} H_{2}+\partial_{2} H_{1}=0,
\end{array}\right.
$$

under the constraint

$$
\partial_{1} H_{1}+\partial_{2} H_{2}=0
$$

on the initial data. From now on we write

$$
\mathbf{H}=\left(H_{1}, H_{2}\right)^{T},
$$

hoping that this small abuse of notation will create no confusion for the reader.

Let us assume that the moving interface $\Gamma(t)$ takes the form

$$
\Gamma(t):=\left\{\left(x_{1}, x_{2}\right) \in \mathbb{R}^{2}, x_{2}=\zeta\left(x_{1}, t\right)\right\},
$$

where $t \in[0, T]$, and that $\Omega^{ \pm}(t)=\left\{x_{2} \gtrless \zeta\left(x_{1}, t\right)\right\}$.

The plasma variables are connected with the vacuum magnetic and electric fields on the interface $\Gamma(t)$ through the relations [4]

$$
\begin{aligned}
& \partial_{t} \zeta=\mathbf{v} \cdot N, \quad q=\frac{1}{2}\left(H_{1}^{2}+H_{2}^{2}-E^{2}\right), \\
& \mathbf{B} \cdot N=0, \quad \mathbf{H} \cdot N=0, \quad E-\nu \zeta_{t} H_{1}=0 \quad \text { on } \quad \Gamma(t),
\end{aligned}
$$

where $N=\left(-\partial_{1} \zeta, 1\right)$ is a normal vector and $[q]=\left.q\right|_{\Gamma}-\frac{1}{2}|\mathbf{H}|_{\mid \Gamma}^{2}+\frac{1}{2}|\mathbf{E}|_{\mid \Gamma}^{2}$ is the jump of the total pressure across the interface.

A stationary solution of the 2-D equations (4) and (6), (7) with interface located at $\left\{x_{2}=0\right\}$ is given by the constant states

$$
\begin{gathered}
\mathbf{v}^{0}=\left(v_{1}^{0}, 0\right)^{T}, \quad \mathbf{B}^{0}=\left(B_{1}^{0}, 0\right)^{T}, \\
\mathbf{H}^{0}=\left(H_{1}^{0}, 0\right)^{T}, \quad E^{0}=0, \quad q^{0}=\frac{1}{2}\left(H_{1}^{0}\right)^{2} .
\end{gathered}
$$

We will consider the propagation of surface waves localized near the interface. The corresponding solutions must satisfy the decay conditions

$$
\begin{aligned}
& \lim _{x_{2} \rightarrow+\infty}(\mathbf{v}, \mathbf{B}, q)=U^{0}:=\left(v_{1}^{0}, 0, B_{1}^{0}, 0, q^{0}\right), \\
& \lim _{x_{2} \rightarrow-\infty}(\mathbf{H}, E)=V^{0}:=\left(H_{1}^{0}, 0,0\right) .
\end{aligned}
$$




\section{The ASYMPtotic EXPANSION}

As in [1] we suppose that the perturbed interface has a slope of the order $\varepsilon$, where $\varepsilon$ is a small parameter. With respect to dimensionless variables in which the wavelength of the perturbation and the velocity of the surface wave are of the order one, the time scale for quadratically nonlinear effects to significantly alter the wave profile is of the order $\varepsilon^{-1}$. We therefore introduce a "slow" time variable $\tau=\varepsilon t$. We also introduce a spatial variable $\theta=x_{1}-\lambda t$ in a reference frame moving with the surface wave. Here, $\lambda$ is the linearized phase velocity of the wave, which we will determine as part of the solution.

We write the perturbed location of the interface as

$$
x_{2}=\varepsilon \varphi(\theta, \tau ; \varepsilon),
$$

and define a new independent variable

$$
\eta=x_{2}-\varepsilon \varphi(\theta, \tau ; \varepsilon),
$$

so that the perturbed interface is located at $\eta=0$. We look for an asymptotic expansion of the solution $U=(\mathbf{v}, \mathbf{B}, q)^{T}, V=(\mathbf{H}, E)^{T}$ and $\varphi$ as $\varepsilon \rightarrow 0$ of the form

$$
\begin{array}{ll}
U(\theta, \eta, \tau ; \varepsilon)=U^{0}+\varepsilon U^{(1)}(\theta, \eta, \tau)+\varepsilon^{2} U^{(2)}(\theta, \eta, \tau)+O\left(\varepsilon^{3}\right), & \eta>0, \\
V(\theta, \eta, \tau ; \varepsilon)=V^{0}+\varepsilon V^{(1)}(\theta, \eta, \tau)+\varepsilon^{2} V^{(2)}(\theta, \eta, \tau)+O\left(\varepsilon^{3}\right), & \eta<0, \\
\varphi(\theta, \tau ; \varepsilon)=\varphi^{(1)}(\theta, \tau)+\varepsilon \varphi^{(2)}(\theta, \tau)+O\left(\varepsilon^{2}\right) . &
\end{array}
$$

We expand the partial derivatives with respect to the original time and space variables as

$$
\begin{aligned}
& \partial_{t}=-\lambda \partial_{\theta}+\varepsilon\left(\partial_{\tau}+\lambda \varphi_{\theta} \partial_{\eta}\right)-\varepsilon^{2} \varphi_{\tau} \partial_{\eta}, \\
& \partial_{x_{1}}=\partial_{\theta}-\varepsilon \varphi_{\theta} \partial_{\eta}, \\
& \partial_{x_{2}}=\partial_{\eta} .
\end{aligned}
$$

We substitute these expansions in (4), (6), Taylor expand the result with respect to $\varepsilon$ and equate coefficients of $\varepsilon^{1}$ and $\varepsilon^{2}$ to zero. In the interior the asymptotic solution satisfies at the first order

$$
\begin{aligned}
& \begin{cases}\left(\lambda-v_{1}^{0}\right) \partial_{\theta} v^{(1)}+B_{1}^{0} \partial_{\theta} B^{(1)}-\left(\begin{array}{c}
\partial_{\theta} \\
\partial_{\eta}
\end{array}\right) q^{(1)}=0, & \\
\left(\lambda-v_{1}^{0}\right) \partial_{\theta} B^{(1)}+B_{1}^{0} \partial_{\theta} v^{(1)}=0, & \text { for } \eta>0, \\
\partial_{\theta} v_{1}^{(1)}+\partial_{\eta} v_{2}^{(1)}=0, & \end{cases} \\
& \qquad \begin{cases}\nu \lambda \partial_{\theta} H_{1}^{(1)}-\partial_{\eta} E^{(1)}=0, \\
\nu \lambda \partial_{\theta} H_{2}^{(1)}+\partial_{\theta} E^{(1)}=0, \\
\nu \lambda \partial_{\theta} E^{(1)}+\partial_{\theta} H_{2}^{(1)}-\partial_{\eta} H_{1}^{(1)}=0, & \text { for } \eta<0 .\end{cases}
\end{aligned}
$$

We expand the jump conditions in (7), with $\zeta=\varepsilon \varphi$, and equate coefficients of $\varepsilon^{1}$ and $\varepsilon^{2}$ to zero. We find that the solutions satisfy at the first order the following jump conditions

$$
\left\{\begin{array}{l}
\left(\lambda-v_{1}^{0}\right) \partial_{\theta} \varphi^{(1)}+v_{2}^{(1)}=0, \\
B_{1}^{0} \partial_{\theta} \varphi^{(1)}-B_{2}^{(1)}=0, \quad H_{1}^{0} \partial_{\theta} \varphi^{(1)}-H_{2}^{(1)}=0, \\
q^{(1)}=H_{1}^{0} H_{1}^{(1)}, \quad E^{(1)}+\nu \lambda H_{1}^{0} \partial_{\theta} \varphi^{(1)}=0, \quad \text { for } \eta=0 .
\end{array}\right.
$$

At the second order we obtain

$$
\begin{aligned}
& \begin{cases}\left(\lambda-v_{1}^{0}\right) \partial_{\theta} v^{(2)}+B_{1}^{0} \partial_{\theta} B^{(2)}-\left(\begin{array}{c}
\partial_{\theta} \\
\partial_{\eta}
\end{array}\right) q^{(2)}=p_{1}, & \\
\left(\lambda-v_{1}^{0}\right) \partial_{\theta} B^{(2)}+B_{1}^{0} \partial_{\theta} v^{(2)}=p_{2}, & \text { for } \eta>0, \\
-\partial_{\theta} v_{1}^{(2)}-\partial_{\eta} v_{2}^{(2)}=p_{3}, & \end{cases} \\
& \qquad \begin{cases}\nu \lambda \partial_{\theta} H_{1}^{(2)}-\partial_{\eta} E^{(2)}=p_{1}^{\prime}, \\
\nu \lambda \partial_{\theta} H_{2}^{(2)}+\partial_{\theta} E^{(2)}=p_{2}^{\prime}, \\
\nu \lambda \partial_{\theta} E^{(2)}+\partial_{\theta} H_{2}^{(2)}-\partial_{\eta} H_{1}^{(2)}=p_{3}^{\prime}, & \text { for } \eta<0,\end{cases}
\end{aligned}
$$


and the jump conditions

$$
\left\{\begin{array}{l}
\left(\lambda-v_{1}^{0}\right) \partial_{\theta} \varphi^{(2)}+v_{2}^{(2)}=r_{1}, \\
B_{1}^{0} \partial_{\theta} \varphi^{(2)}-B_{2}^{(2)}=r_{2}, \quad H_{1}^{0} \partial_{\theta} \varphi^{(2)}-H_{2}^{(2)}=r_{3}, \\
q^{(2)}-H_{1}^{0} H_{1}^{(2)}=r_{4}, \quad E^{(2)}+\nu \lambda H_{1}^{0} \partial_{\theta} \varphi^{(2)}=r_{5}, \quad \text { for } \eta=0,
\end{array}\right.
$$

where we have denoted

$$
\begin{gathered}
p_{1}:=\left(\partial_{\tau}+\lambda \varphi_{\theta}^{(1)} \partial_{\eta}\right) v^{(1)}+\left(v_{1}^{(1)} \partial_{\theta}+v_{2}^{(1)} \partial_{\eta}-v_{1}^{0} \varphi_{\theta}^{(1)} \partial_{\eta}\right) v^{(1)} \\
-\left(B_{1}^{(1)} \partial_{\theta}+B_{2}^{(1)} \partial_{\eta}-B_{1}^{0} \varphi_{\theta}^{(1)} \partial_{\eta}\right) B^{(1)}-\left(\begin{array}{c}
\varphi_{\theta}^{(1)} \partial_{\eta} q^{(1)} \\
0
\end{array}\right), \\
p_{2}:=\left(\partial_{\tau}+\lambda \varphi_{\theta}^{(1)} \partial_{\eta}\right) B^{(1)}+\left(v_{1}^{(1)} \partial_{\theta}+v_{2}^{(1)} \partial_{\eta}-v_{1}^{0} \varphi_{\theta}^{(1)} \partial_{\eta}\right) B^{(1)} \\
\quad-\left(B_{1}^{(1)} \partial_{\theta}+B_{2}^{(1)} \partial_{\eta}-B_{1}^{0} \varphi_{\theta}^{(1)} \partial_{\eta}\right) v^{(1)}, \\
p_{3}:=-\varphi_{\theta}^{(1)} \partial_{\eta} v_{1}^{(1)}, \quad p_{2}^{\prime}:=\nu\left(\partial_{\tau}+\lambda \varphi_{\theta}^{(1)} \partial_{\eta}\right) H_{2}^{(1)}+\varphi_{\theta}^{(1)} \partial_{\eta} E^{(1)}, \\
p_{1}^{\prime}:=\nu\left(\partial_{\tau}+\lambda \varphi_{\theta}^{(1)} \partial_{\eta}\right) H_{1}^{(1)}, \quad r_{2}:=-B_{1}^{(1)} \partial_{\theta} \varphi^{(1)}, \\
p_{3}^{\prime}:=\nu\left(\partial_{\tau}+\lambda \varphi_{\theta}^{(1)} \partial_{\eta}\right) E^{(1)}+\varphi_{\theta}^{(1)} \partial_{\eta} H_{2}^{(1)}, \\
r_{1}:=\left(\partial_{\tau}+v_{1}^{(1)} \partial_{\theta}\right) \varphi^{(1)}, \quad r_{4}:=\frac{1}{2}\left(\left|H^{(1)}\right|^{2}-\left(E^{(1)}\right)^{2}\right), \\
r_{3}:=-H_{1}^{(1)} \partial_{\theta} \varphi^{(1)}, \\
r_{5}:=-\nu \lambda H_{1}^{(1)} \partial_{\theta} \varphi^{(1)}+\nu H_{1}^{0} \partial_{\tau} \varphi^{(1)} .
\end{gathered}
$$

The next step is the resolution of equations (10)-(15).

\section{THE FIRST ORDER EQUATIONS}

We first solve system (10)-(12) by Fourier transformation. Introducing the Fourier transforms

$$
\begin{aligned}
\hat{U}^{(1)}(k, \eta, \tau) & =\frac{1}{2 \pi} \int_{-\infty}^{+\infty} U^{(1)}(\theta, \eta, \tau) e^{-i k \theta} d \theta \\
\hat{V}^{(1)}(k, \eta, \tau) & =\frac{1}{2 \pi} \int_{-\infty}^{+\infty} V^{(1)}(\theta, \eta, \tau) e^{-i k \theta} d \theta \\
\hat{\varphi}^{(1)}(k, \tau) & =\frac{1}{2 \pi} \int_{-\infty}^{+\infty} \varphi^{(1)}(\theta, \tau) e^{-i k \theta} d \theta
\end{aligned}
$$

and Fourier transforming (10)-(12) with respect to $\theta$, we find a system of the form

$$
i k \mathcal{A} \hat{U}^{(1)}+\mathcal{B} \partial_{\eta} \hat{U}^{(1)}=0
$$

with suitable real symmetric matrices $\mathcal{A}, \mathcal{B}$. As shown in [11], the general solution of (16) is

$$
\hat{U}^{(1)}(k, \eta, \tau)=a(k, \tau) e^{-k \eta} \mathbf{R}+b(k, \tau) e^{k \eta} \overline{\mathbf{R}}, \quad \eta>0
$$

where

$$
\mathbf{R}=\left(\lambda-v_{1}^{0}, i\left(\lambda-v_{1}^{0}\right),-B_{1}^{0},-i B_{1}^{0}, d\right)^{T} \quad \text { and } \quad d:=\left(\lambda-v_{1}^{0}\right)^{2}-\left(B_{1}^{0}\right)^{2} .
$$

Here $a(k, \tau)$ and $b(k, \tau)$ are arbitrary complex-valued functions, the bar denotes a complex conjugate. The condition (8) at infinity implies

$$
\lim _{\eta \rightarrow+\infty} \hat{U}^{(1)}(k, \eta, \tau)=0
$$

yielding from (17)

$$
\hat{U}^{(1)}(k, \eta, \tau)= \begin{cases}a(k, \tau) e^{-k \eta} \mathbf{R}, & \text { if } k>0, \\ b(k, \tau) e^{k \eta} \overline{\mathbf{R}}, & \text { if } k<0 .\end{cases}
$$

Then we consider problem (11) for $\eta<0$. First of all we see that $\hat{E}^{(1)}$ obeys the equation

$$
\partial_{\eta}^{2} \hat{E}^{(1)}+k^{2}\left(\nu^{2} \lambda^{2}-1\right) \hat{E}^{(1)}=0 .
$$


Thus, in order to have

$$
\lim _{\eta \rightarrow-\infty} \hat{V}^{(1)}(k, \eta, \tau)=0
$$

(obtained from (8)), we need to prescribe in (20)

$$
\nu|\lambda|<1
$$

It is easily seen that the general solution of (11) is

$$
\begin{aligned}
& \hat{H}_{1}^{(1)}(k, \eta, \tau)=\frac{\sigma(\lambda)}{i \nu \lambda}\left\{\alpha(k, \tau) e^{\sigma(\lambda) k \eta}-\beta(k, \tau) e^{-\sigma(\lambda) k \eta}\right\}, \\
& \hat{H}_{2}^{(1)}(k, \eta, \tau)=-\frac{1}{\nu \lambda}\left\{\alpha(k, \tau) e^{\sigma(\lambda) k \eta}+\beta(k, \tau) e^{-\sigma(\lambda) k \eta}\right\} \\
& \hat{E}^{(1)}(k, \eta, \tau)=\alpha(k, \tau) e^{\sigma(\lambda) k \eta}+\beta(k, \tau) e^{-\sigma(\lambda) k \eta},
\end{aligned}
$$

where $\alpha(k, \tau)$ and $\beta(k, \tau)$ are arbitrary complex-valued functions and

$$
\sigma(\lambda):=\sqrt{1-\nu^{2} \lambda^{2}}>0 .
$$

Finally, imposing the condition (21) at infinity to (23) we find that

$$
\hat{V}^{(1)}(k, \eta, \tau)= \begin{cases}\alpha(k, \tau) e^{\sigma(\lambda) k \eta}\left(\begin{array}{c}
-i \sigma(\lambda) / \nu \lambda \\
-1 / \nu \lambda \\
1
\end{array}\right), & \text { if } k>0, \\
\beta(k, \tau) e^{-\sigma(\lambda) k \eta}\left(\begin{array}{c}
i \sigma(\lambda) / \nu \lambda \\
-1 / \nu \lambda \\
1
\end{array}\right), & \text { if } k<0 .\end{cases}
$$

Next, we use the solution (19), (24) in the jump conditions (12). First we consider the case $k>0$. Under the assumption $\lambda-v_{1}^{0} \neq 0$ or $B_{1}^{0} \neq 0$, the resulting equations may be written as a linear system for the unknowns $\left(a, \alpha, k \hat{\varphi}^{(1)}\right)$ :

$$
\left(\begin{array}{ccc}
1 & 0 & 1 \\
0 & 1 & i \nu \lambda H_{1}^{0} \\
d & i \sigma(\lambda) H_{1}^{0} / \nu \lambda & 0
\end{array}\right)\left(\begin{array}{c}
a \\
\alpha \\
k \hat{\varphi}^{(1)}
\end{array}\right)=0 .
$$

This system has a nontrivial solution if and only if

$$
d=\left(\lambda-v_{1}^{0}\right)^{2}-\left(B_{1}^{0}\right)^{2}=\left(H_{1}^{0}\right)^{2} \sigma(\lambda)
$$

Real roots $\lambda$ of (22), (26) do not exist for all stationary states $\left(v_{1}^{0}, B_{1}^{0}, H_{1}^{0}\right)$, but only for those satisfying a suitable physical condition, as discussed in the following lemma.

Lemma 1. (1) If $\left|B_{1}^{0}\right|>\left|v_{1}^{0}\right|+1 / \nu$, equation (26) does not have any real root.

(2) If $\left|B_{1}^{0}\right|=\left|v_{1}^{0}\right|+1 / \nu$, for all $\left|H_{1}^{0}\right|>0$ and $v_{1}^{0} \neq 0$ there exists one real root $\lambda=-\operatorname{sgn}\left(\mathrm{v}_{1}^{0}\right) / \nu$. If $v_{1}^{0}=0$ then $\lambda= \pm 1 / \nu$. Thus in any case $|\lambda|=1 / \nu$.

(3) If $\left|v_{1}^{0}\right|-1 / \nu \leq\left|B_{1}^{0}\right|<\left|v_{1}^{0}\right|+1 / \nu$, for all $\left|H_{1}^{0}\right|>0$ there exist one or two real roots $\lambda$ of (26) such that $|\lambda|<1 / \nu$.

(4) If $\left|B_{1}^{0}\right|<\left|v_{1}^{0}\right|-1 / \nu$, there exists $H^{*}>0$ such that, for all $\left|H_{1}^{0}\right| \geq H^{*}$, there exist two real roots $\lambda$ of (26) such that $|\lambda|<1 / \nu$ (coincident roots if $\left|H_{1}^{0}\right|=H^{*}$ ); if $\left|H_{1}^{0}\right|<H^{*}$ (26) does not have any real root.

Proof. See [11].

We choose $\lambda$ to be one of the values found in Lemma 1 such that $|\lambda|<1 / \nu$ (cases 3 and 4 ). Observe that for all such $|\lambda|<1 / \nu$, from (26) there holds $\lambda \neq v_{1}^{0}$ and $\lambda \neq v_{1}^{0} \pm B_{1}^{0}$, i.e. $d \neq 0$.

The solution of $(25)$ is then

$$
a=-k \hat{\varphi}^{(1)}, \quad \alpha=-\nu \lambda H_{1}^{0} i k \hat{\varphi}^{(1)} \quad \text { if } k>0 .
$$

For $k<0$ the analysis is similar and gives the solution

$$
b=k \hat{\varphi}^{(1)}, \quad \beta=-\nu \lambda H_{1}^{0} i k \hat{\varphi}^{(1)} \quad \text { if } k<0,
$$


under the same condition (26).

Summarizing these results, we have shown that when $\lambda$ satisfies (22), (26), the solution of (10)-(12), (18), (21) is given by

$$
\begin{gathered}
\hat{U}^{(1)}(k, \eta, \tau)= \begin{cases}-|k| \hat{\varphi}^{(1)}(k, \tau) e^{-k \eta} \mathbf{R}, & \text { if } k>0, \\
-|k| \hat{\varphi}^{(1)}(k, \tau) e^{k \eta} \overline{\mathbf{R}}, & \text { if } k<0,\end{cases} \\
\hat{V}^{(1)}(k, \eta, \tau)=H_{1}^{0} \hat{\varphi}^{(1)}(k, \tau) e^{\sigma(\lambda)|k| \eta}\left(\begin{array}{c}
-\sigma(\lambda)|k| \\
i k \\
-i \nu \lambda k
\end{array}\right) .
\end{gathered}
$$

This solution depends on the unknown function $\hat{\varphi}^{(1)}(k, \tau)$, which describes the profile of the surface wave, and will be determined through a solvability condition for the equations for the second order corrections.

\section{THE SECOND ORDER EQUATIONS}

We introduce the Fourier transforms

$$
\begin{aligned}
\hat{U}^{(2)}(k, \eta, \tau) & =\frac{1}{2 \pi} \int_{-\infty}^{+\infty} U^{(2)}(\theta, \eta, \tau) e^{-i k \theta} d \theta \\
\hat{V}^{(2)}(k, \eta, \tau) & =\frac{1}{2 \pi} \int_{-\infty}^{+\infty} V^{(2)}(\theta, \eta, \tau) e^{-i k \theta} d \theta \\
\hat{\varphi}^{(2)}(k, \tau) & =\frac{1}{2 \pi} \int_{-\infty}^{+\infty} \varphi^{(2)}(\theta, \tau) e^{-i k \theta} d \theta .
\end{aligned}
$$

5.1. The second order equations in the plasma region. Fourier transforming (13) with respect to $\theta$ gives the system of equations

$$
i k \mathcal{A} \hat{U}^{(2)}+\mathcal{B} \partial_{\eta} \hat{U}^{(2)}=\hat{p},
$$

where $\mathcal{A}, \mathcal{B}$ are the same matrices of (16). From (8), the solution of (31) must satisfy the decay condition

$$
\lim _{\eta \rightarrow+\infty} \hat{U}^{(2)}(k, \eta, \tau)=0 .
$$

In order to solve (31), (32), in [11] we proceed as in [1]. We look for a solution of (31) in the form

$$
\hat{U}^{(2)}(k, \eta, \tau)=\mathbf{S}(k, \eta, \tau)+a(k, \eta, \tau) \mathbf{R}+b(k, \eta, \tau) \overline{\mathbf{R}}
$$

with a suitable vector-valued function $\mathbf{S}$ and scalar functions $a, b$. We look for $\mathbf{S}$ such that

$$
\mathbf{L} \cdot \mathcal{B S}=\overline{\mathbf{L}} \cdot \mathcal{B S}=0
$$

where $\mathbf{L}$ is an eigenvector of

$$
\mathbf{L} \cdot(i \mathcal{A}-\mathcal{B})=0
$$

normalized by

$$
\mathbf{L} \cdot \mathcal{B R}=\overline{\mathbf{L}} \cdot \mathcal{B} \overline{\mathbf{R}}=1
$$

that is

$$
\mathbf{L}=-\frac{1}{2 i d\left(\lambda-v_{1}^{0}\right)} \mathbf{R} .
$$

In [11] we show that the solution $\mathbf{S}$ is given by

$$
\mathbf{S}=\left(\frac{1}{k} \mathbf{L}_{1} \cdot \hat{p}, 0, \frac{1}{k} \mathbf{L}_{3} \cdot \hat{p}, \frac{1}{k} \mathbf{L}_{4} \cdot \hat{p}, 0\right)^{T},
$$

where

$$
\begin{gathered}
\mathbf{L}_{1}=\frac{1}{d}\left(-i\left(\lambda-v_{1}^{0}\right), 0, i B_{1}^{0}, 0,0\right)^{T}, \quad \mathbf{L}_{3}=\frac{1}{d}\left(i B_{1}^{0}, 0,-i\left(\lambda-v_{1}^{0}\right), 0,0\right)^{T} \\
\mathbf{L}_{4}=\left(0,0,0,-\frac{i}{\lambda-v_{1}^{0}}, 0\right)^{T} .
\end{gathered}
$$


As for $a, b$, they are given by

$$
\begin{aligned}
& a(k, \eta, \tau)=e^{-k \eta}\left(a_{0}(k, \tau)+\int_{0}^{\eta} \mathbf{L} \cdot \hat{p}\left(k, \eta^{\prime}, \tau\right) e^{k \eta^{\prime}} d \eta^{\prime}\right) \\
& b(k, \eta, \tau)=e^{k \eta}\left(b_{0}(k, \tau)+\int_{0}^{\eta} \overline{\mathbf{L}} \cdot \hat{p}\left(k, \eta^{\prime}, \tau\right) e^{-k \eta^{\prime}} d \eta^{\prime}\right)
\end{aligned}
$$

where $a_{0}(k, \tau), b_{0}(k, \tau)$ are arbitrary functions of integration, that will be chosen later.

In order to verify the decay condition $(32)$ for $\hat{U}^{(2)}(k, \eta, \tau)$, we first explicitly calculate $\mathbf{S}(k, \eta, \tau)$ from (35). This is a very long expression that we don't report for the sake of brevity. The fact that we need here is that $\mathbf{S}$ depends on $\eta$ only through the exponentials of $-|k| \eta$ and $-(|k-\ell|+|\ell|) \eta$, so that

$$
\lim _{\eta \rightarrow+\infty} \mathbf{S}(k, \eta, \tau)=0
$$

Thus $\hat{U}^{(2)}(k, \eta, \tau)$ satisfies (32) if and only if

$$
\begin{aligned}
& \lim _{\eta \rightarrow+\infty} a(k, \eta, \tau)=0, \\
& \lim _{\eta \rightarrow+\infty} b(k, \eta, \tau)=0 .
\end{aligned}
$$

From (36), (37), and the explicit calculation of $\mathbf{L} \cdot \hat{p}(k, \eta, \tau), \overline{\mathbf{L}} \cdot \hat{p}(k, \eta, \tau)$, it follows that condition (38) is automatically satisfied if $k>0$, and (39) is automatically satisfied if $k<0$. It follows that $a_{0}$ remains undetermined for $k>0$, and $b_{0}$ remains undetermined for $k<0$. Instead, (36)-(39) may be used to determine $a_{0}$ if $k<0$, and $b_{0}$ if $k>0$, as functions of $\hat{\varphi}^{(1)}$ :

$$
\begin{array}{ll}
a_{0}(k, \tau)=-\int_{0}^{+\infty} \mathbf{L} \cdot \hat{p}\left(k, \eta^{\prime}, \tau\right) e^{k \eta^{\prime}} d \eta^{\prime}, & \text { if } k<0, \\
b_{0}(k, \tau)=-\int_{0}^{+\infty} \overline{\mathbf{L}} \cdot \hat{p}\left(k, \eta^{\prime}, \tau\right) e^{-k \eta^{\prime}} d \eta^{\prime}, & \text { if } k>0 .
\end{array}
$$

5.2. The second order equations in vacuum. We take the Fourier transform of (14) in $\theta$ for $\eta<0$. The problem is easily solved by substitution of $i k \hat{H}_{2}^{(2)}=\left(\hat{p}_{2}^{\prime}-i k \hat{E}^{(2)}\right) / \nu \lambda$ in the other equations to give

$$
\partial_{\eta}^{2} \hat{E}^{(2)}+k^{2}\left(\nu^{2} \lambda^{2}-1\right) \hat{E}^{(2)}=-P
$$

where $P=\nu \lambda i k \hat{p}_{3}^{\prime}-i k \hat{p}_{2}^{\prime}+\partial_{\eta} \hat{p}_{1}^{\prime}$. We solve (42) with the decay condition

$$
\lim _{\eta \rightarrow-\infty} \hat{E}^{(2)}(k, \eta, \tau)=0
$$

then we substitute in the other equations to find the other components of $\hat{V}^{(2)}(k, \eta, \tau)$ and obtain

$$
\begin{gathered}
\hat{V}^{(2)}(k, \eta, \tau)=\alpha^{\prime}(k, \tau) e^{\sigma(\lambda) k \eta}\left(\begin{array}{c}
\frac{\sigma(\lambda)}{i \nu \lambda} \\
-\frac{1}{\nu \lambda} \\
1
\end{array}\right) \\
+\frac{1}{2|k| \sigma(\lambda)}\left(\begin{array}{c}
\frac{1}{\nu \lambda i k}\left\{\int_{-\infty}^{0} e^{-\sigma(\lambda)|k||\eta-\zeta|} \partial_{\zeta} P(k, \zeta, \tau) d \zeta-e^{-\sigma(\lambda)|k \eta|} P(k, 0, \tau)\right\} \\
-\frac{1}{\nu \lambda} \int_{-\infty}^{0} e^{-\sigma(\lambda)|k||\eta-\zeta|} P(k, \zeta, \tau) d \zeta \\
\int_{-\infty}^{0} e^{-\sigma(\lambda)|k||\eta-\zeta|} P(k, \zeta, \tau) d \zeta
\end{array}\right)+\left(\begin{array}{c}
\frac{1}{\nu \lambda i k} \hat{p}_{1}^{\prime} \\
\frac{1}{\nu \lambda i k} \hat{p}_{2}^{\prime} \\
0
\end{array}\right), \quad \text { if } k>0,
\end{gathered}
$$




$$
\begin{gathered}
\hat{V}^{(2)}(k, \eta, \tau)=\beta^{\prime}(k, \tau) e^{-\sigma(\lambda) k \eta}\left(\begin{array}{c}
-\frac{\sigma(\lambda)}{i \nu \lambda} \\
-\frac{1}{\nu \lambda} \\
1
\end{array}\right) \\
+\frac{1}{2|k| \sigma(\lambda)}\left(\begin{array}{c}
\frac{1}{\nu \lambda i k}\left\{\int_{-\infty}^{0} e^{-\sigma(\lambda)|k||\eta-\zeta|} \partial_{\zeta} P(k, \zeta, \tau) d \zeta-e^{-\sigma(\lambda)|k \eta|} P(k, 0, \tau)\right\} \\
-\frac{1}{\nu \lambda} \int_{-\infty}^{0} e^{-\sigma(\lambda)|k||\eta-\zeta|} P(k, \zeta, \tau) d \zeta \\
\int_{-\infty}^{0} e^{-\sigma(\lambda)|k||\eta-\zeta|} P(k, \zeta, \tau) d \zeta
\end{array}\right)+\left(\begin{array}{c}
\frac{1}{\nu \lambda \lambda^{i k}} \hat{p}_{1}^{\prime} \\
\frac{\nu \lambda i k}{\hat{p}_{2}^{\prime}} \\
0
\end{array}\right), \quad \text { if } k<0 .
\end{gathered}
$$

Notice that we need to determine the arbitrary functions $\alpha^{\prime}(k, \tau)$ if $k>0$, and $\beta^{\prime}(k, \tau)$ if $k<0$.

5.3. The second order jump conditions. The first-order solution depends on the unknown function $\hat{\varphi}^{(1)}(k, \tau)$, which describes the profile of the surface wave, while the second order solution depends, in addition, on unknown functions $a_{0}(k, \tau), b_{0}(k, \tau)$ and $\alpha^{\prime}(k, \tau), \beta^{\prime}(k, \tau)$. Now we study the second order jump conditions and show that they reduce to a singular linear system of algebraic equations for $\left(a_{0}, b_{0}, \alpha^{\prime}, \beta^{\prime}, \hat{\varphi}^{(2)}\right)$, where $\hat{\varphi}^{(2)}(k, \tau)$ is the Fourier transform of the second-order displacement of the interface. Imposing solvability conditions on this system gives the evolution equation for the function $\hat{\varphi}^{(1)}(k, \tau)$ that we seek.

We take the Fourier transform of (15), where we substitute the second order corrections obtained in the previous sections, evaluated at $\eta=0$.

Let us first assume $k>0$, recalling that in this case we need to determine $a_{0}(k, \tau), \alpha^{\prime}(k, \tau)$ and $\hat{\varphi}^{(2)}(k, \tau)$ (for $\left.k>0\right)$. In this case we obtain the linear system

$$
\left(\begin{array}{ccc}
1 & 0 & 1 \\
1 & 0 & 1 \\
0 & 1 / \nu \lambda & i H_{1}^{0} \\
d & i \sigma(\lambda) H_{1}^{0} / \nu \lambda & 0 \\
0 & 1 & i \nu \lambda H_{1}^{0}
\end{array}\right)\left(\begin{array}{c}
a_{0} \\
\alpha^{\prime} \\
k \hat{\varphi}^{(2)}
\end{array}\right)=\left(\begin{array}{c}
\hat{r}_{1}^{\prime} \\
\hat{r}_{2}^{\prime} \\
\hat{r}_{3}^{\prime} \\
\hat{r}_{4}^{\prime} \\
\hat{r}_{5}^{\prime}
\end{array}\right),
$$

where $\hat{r}_{1}^{\prime}, \ldots, \hat{r}_{5}^{\prime}$ depend on the first order corrections and $b_{0}$ given by (41) (in case $k>0$ it is known). First of all we see that the first two lines of the matrix in the left-hand side of (45) are equal, and we can also verify that $\hat{r}_{1}^{\prime}=\hat{r}_{2}^{\prime}$. Moreover, the last row of the matrix in (45) equals the third one multiplied by $\nu \lambda$, and actually one verifies that $\hat{r}_{5}^{\prime}=\nu \lambda \hat{r}_{3}^{\prime}$. Thus (45) may be reduced to

$$
\left(\begin{array}{ccc}
1 & 0 & 1 \\
0 & 1 / \nu \lambda & i H_{1}^{0} \\
d & i \sigma(\lambda) H_{1}^{0} / \nu \lambda & 0
\end{array}\right)\left(\begin{array}{c}
a_{0} \\
\alpha^{\prime} \\
k \hat{\varphi}^{(2)}
\end{array}\right)=\left(\begin{array}{c}
\hat{r}_{1}^{\prime} \\
\hat{r}_{3}^{\prime} \\
\hat{r}_{4}^{\prime}
\end{array}\right) .
$$

The determinant of the matrix of this system is zero because of (26), i.e. the equation defining $\lambda$. It is easily seen that the rank of this matrix is 2 . Then, the linear system (46) is solvable if and only if the rank of the augmented matrix is also equal to 2, and this is true if the following condition holds:

$$
d \hat{r}_{3}^{\prime}+i H_{1}^{0} \hat{r}_{4}^{\prime}-i H_{1}^{0} d \hat{r}_{1}^{\prime}=0 .
$$

Developing the terms in (47) we get the solvability condition

$$
\left(2 \frac{\lambda-v_{1}^{0}}{d}+\frac{\nu^{2} \lambda}{\sigma(\lambda)^{2}}\right) \hat{\varphi}_{\tau}^{(1)}(k, \tau)+i \int_{-\infty}^{+\infty} \Lambda_{+}(k, \ell) \hat{\varphi}^{(1)}(k-\ell, \tau) \hat{\varphi}^{(1)}(\ell, \tau) d \ell=0, \quad k>0,
$$

where we have denoted

$$
\begin{aligned}
\Lambda_{+}(k, \ell)=\ell \frac{|k-\ell|(|k-\ell|-|\ell|)+(k-\ell)|\ell|-|k-\ell| \ell}{|k-\ell|+|k|+|\ell|} & +\frac{(k-\ell)|\ell|(|\ell|-\ell)}{|k|+|\ell|}-(k-\ell)|\ell| \\
& +\sigma(\lambda)\left\{-k|\ell|+\frac{1}{2}((k+\ell) \ell-|k-\ell||\ell|)\right\} .
\end{aligned}
$$

Thus, when $k>0$, the system (46) is solvable if and only if $\hat{\varphi}^{(1)}$ satisfies equation (48) and then the rank of the augmented matrix of the system is equal to 2. Given the solution $\hat{\varphi}^{(1)}$ of (48) we compute $\hat{U}^{(1)}, \hat{V}^{(1)}$ from (29), (30). Thus the leading-order term of the asymptotic expansion is uniquely 
determined. From system (46) we may obtain $a_{0}, \alpha^{\prime}$ in terms of an arbitrary second order wave profile $\hat{\varphi}^{(2)}$, and in turn $\hat{U}^{(2)}, \hat{V}^{(2)}$ from the expressions obtained in the previous sections. The wave profile $\hat{\varphi}^{(2)}$ should be determined by considering higher order terms of the asymptotic expansion, see [10].

The analysis for $k<0$ is similar and leads to the solvability condition

$$
\left(2 \frac{\lambda-v_{1}^{0}}{d}+\frac{\nu^{2} \lambda}{\sigma(\lambda)^{2}}\right) \hat{\varphi}_{\tau}^{(1)}(k, \tau)+i \int_{-\infty}^{+\infty} \Lambda_{-}(k, \ell) \hat{\varphi}^{(1)}(k-\ell, \tau) \hat{\varphi}^{(1)}(\ell, \tau) d \ell=0, \quad k<0,
$$

where we have denoted

$$
\begin{aligned}
\Lambda_{-}(k, \ell)=\ell \frac{|k-\ell|(|k-\ell|-|\ell|)-(k-\ell)|\ell|+|k-\ell| \ell}{|k-\ell|+|k|+|\ell|} & +\frac{(k-\ell)|\ell|(|\ell|+\ell)}{|k|+|\ell|}-(k-\ell)|\ell| \\
& +\sigma(\lambda)\left\{-k|\ell|-\frac{1}{2}((k+\ell) \ell-|k-\ell||\ell|)\right\} .
\end{aligned}
$$

Given the solution $\hat{\varphi}^{(1)}$ of (50) we compute $\hat{U}^{(1)}, \hat{V}^{(1)}$ from (29), (30). We may get $b_{0}, \beta^{\prime}$ in terms of an arbitrary second order wave profile $\hat{\varphi}^{(2)}$, and in turn $\hat{U}^{(2)}, \hat{V}^{(2)}$ from the expressions obtained in the previous sections. Again, also for $k<0$ the wave profile $\hat{\varphi}^{(2)}$ should be determined by considering higher order terms of the asymptotic expansion, see [10].

5.4. The kernel. The equations (48), (50) can be written in more compact form as

$$
\left(2 \frac{\lambda-v_{1}^{0}}{d}+\frac{\nu^{2} \lambda}{\sigma(\lambda)^{2}}\right) \hat{\varphi}_{\tau}^{(1)}(k, \tau)+i \int_{-\infty}^{+\infty} \Lambda_{0}(k, \ell) \hat{\varphi}^{(1)}(k-\ell, \tau) \hat{\varphi}^{(1)}(\ell, \tau) d \ell=0, \quad \forall k \neq 0,
$$

with kernel

$$
\Lambda_{0}(k, \ell)= \begin{cases}\Lambda_{+}(k, \ell) & k>0, \\ \Lambda_{-}(k, \ell) & k<0,\end{cases}
$$

where $\Lambda_{ \pm}$are defined in (49), (51). This form is not convenient and so we look for a different formula. First we write it as

$$
\Lambda_{0}(k, \ell)=\operatorname{sgn}(\mathrm{k}) \tilde{\Lambda}_{0}(\mathrm{k}-\ell, \ell),
$$

so that the integral in (52) takes the form of a convolution product. Moreover, the new kernel $\tilde{\Lambda}_{0}(k, \ell)$ can be equivalently replaced in (52) by the symmetrized kernel

$$
\tilde{\Lambda}(k, \ell)=\frac{1}{2}\left(\tilde{\Lambda}_{0}(k, \ell)+\tilde{\Lambda}_{0}(\ell, k)\right),
$$

because the antisymmetric part of $\tilde{\Lambda}_{0}$ gives a vanishing integral. Thus we can write (52) as

$$
\left(2 \frac{\lambda-v_{1}^{0}}{d}+\frac{\nu^{2} \lambda}{\sigma(\lambda)^{2}}\right) \hat{\varphi}_{\tau}^{(1)}(k, \tau)+i \operatorname{sgn}(\mathrm{k}) \int_{-\infty}^{+\infty} \tilde{\Lambda}(\mathrm{k}-\ell, \ell) \hat{\varphi}^{(1)}(\mathrm{k}-\ell, \tau) \hat{\varphi}^{(1)}(\ell, \tau) \mathrm{d} \ell=0, \quad \forall \mathrm{k} \neq 0
$$

The explicit formula of $\tilde{\Lambda}$ is still rather complicated, see [11], but there is the way to simplify it. First of all we verify that $\tilde{\Lambda}$ satisfies the following properties

$$
\begin{array}{lll}
\tilde{\Lambda}(k, \ell)=\tilde{\Lambda}(\ell, k) & & \text { (symmetry), } \\
\tilde{\Lambda}(k, \ell)=\tilde{\Lambda}(-k,-\ell) & & \text { (reality), } \\
\tilde{\Lambda}(\alpha k, \alpha \ell)=\alpha^{2} \tilde{\Lambda}(k, \ell) & \forall \alpha>0 & \text { (homogeneity). }
\end{array}
$$

Considering some particular cases we can considerably simplify $\tilde{\Lambda}$ as follows

$$
\tilde{\Lambda}(k, \ell)= \begin{cases}-(1+\sigma(\lambda)) k \ell & \text { if } k>0, \ell>0, \\ (1+\sigma(\lambda)) \ell(k+\ell) & \text { if } k+\ell>0, \ell<0 .\end{cases}
$$

Then the values of $\tilde{\Lambda}$ in the other regions of the $(k, \ell)$-plane follow from $(55),(56)$. Finally $\tilde{\Lambda}$ can be written in a different way as

$$
\tilde{\Lambda}(k, \ell)=-(1+\sigma(\lambda)) \frac{2|k+\ell||k||\ell|}{|k+\ell|+|k|+|\ell|},
$$


and, after an appropriate rescaling in time, we write (54), (57) as

$$
\hat{\varphi}_{\tau}^{(1)}(k, \tau)+i \operatorname{sgn}(\mathrm{k}) \int_{-\infty}^{+\infty} \Lambda(\mathrm{k}-\ell, \ell) \hat{\varphi}^{(1)}(\mathrm{k}-\ell, \tau) \hat{\varphi}^{(1)}(\ell, \tau) \mathrm{d} \ell=0, \quad \forall \mathrm{k} \neq 0,
$$

with the new kernel $\Lambda$ defined by

$$
\Lambda(k, \ell)=\frac{2|k+\ell||k||\ell|}{|k+\ell|+|k|+|\ell|} .
$$

The spacial form of $(58),(59)$ is, see $[2,5]$,

$$
\varphi_{\tau}^{(1)}+\frac{1}{2} \mathbb{H}\left[\Phi^{2}\right]_{\theta \theta}+\Phi \varphi_{\theta \theta}^{(1)}=0, \quad \Phi=\mathbb{H}\left[\varphi^{(1)}\right],
$$

where $\mathbb{H}$ denotes the Hilbert transform. After renaming of variables it becomes (1), (2).

Equation (58), (59) is well-known as it coincides with the amplitude equation for nonlinear Rayleigh waves [5] and describes the propagation of surface waves on a tangential discontinuity (current-vortex sheet) in incompressible MHD [1]. The derivation of the asymptotic equation (58), (59) also for the plasma-interface problem confirms that it is a canonical model equation for nonlinear surface wave solutions of hyperbolic conservation laws, analogous to the inviscid Burgers equation for bulk waves.

$\Lambda$, defined in (59), is perhaps the simplest kernel arising for surface waves. It satisfies the properties

$$
\begin{aligned}
& \Lambda(k, \ell)=\Lambda(\ell, k) \quad \text { (symmetry), } \\
& \Lambda(k, \ell)=\overline{\Lambda(-k,-\ell)} \quad \text { (reality), } \\
& \Lambda(\alpha k, \alpha \ell)=\alpha^{2} \Lambda(k, \ell) \quad \forall \alpha>0 \quad \text { (homogeneity), } \\
& \Lambda(k+\ell,-\ell)=\overline{\Lambda(k, \ell)} \quad \forall k, \ell \in \mathbb{R} \quad \text { (Hamiltonian). }
\end{aligned}
$$

The value 2 of the scaling exponent in (60c) is consistent with the dimensional analysis in [2] for surface waves. It is shown by Alì et al. [2] that (60d) is a sufficient condition for (54), in addition to (60a), (60b), to admit a Hamiltonian structure, see also [5, 6].

The results of Sections 3 to 5 are summarized in the following theorem.

Theorem 2. Assume that $v_{1}^{0}, B_{1}^{0}, H_{1}^{0}$ are as in (3) or (4) of Lemma 1, and let $\lambda$ be a real root of (26). Then the solution $U=(\mathbf{v}, \mathbf{B}, q)^{T}, V=(\mathbf{H}, E)^{T}, \varphi$ of (4), (6), (7) admits the asymptotic expansion (9), where the first order terms of the expansion are defined in (29), (30). The location of the plasma-vacuum interface is given by

$$
x_{2}=\varepsilon \varphi^{(1)}\left(x_{1}-\lambda t, \varepsilon t\right)+O\left(\varepsilon^{2}\right),
$$

as $\varepsilon \rightarrow 0$, with $t=O\left(\varepsilon^{-1}\right)$ and $\lambda$ the linearized phase velocity of the surface wave. After an appropriate rescaling in time, the Fourier transform of the leading order perturbation $\varphi^{(1)}(\theta, \tau)$ satisfies the amplitude equation (58), (59).

We wish to stress that for the existence of surface waves propagating on the plasma-vacuum interface, it is necessary to have a real root $\lambda$ of (26) satisfying (22). This is obtained if the basic state $v_{1}^{0}, B_{1}^{0}, H_{1}^{0}$ is as in (3) or (4) of Lemma 1.

\section{NONCANONICAL VARIABLES AND WELL-POSEDNESS}

As in [7] we introduce the noncanonical dependent variable $\psi(\theta, \tau)$ defined by

$$
\psi(\theta, \tau)=\left|\partial_{\theta}\right|^{1 / 2} \varphi^{(1)}(\theta, \tau), \quad \hat{\psi}(k, \tau)=|k|^{1 / 2} \hat{\varphi}^{(1)}(k, \tau)
$$

Then rewriting equation (58) in terms of $\psi$ gives

$$
\hat{\psi}_{\tau}(k, \tau)+i k \int_{-\infty}^{+\infty} S(k-\ell, \ell) \hat{\psi}(k-\ell, \tau) \hat{\psi}(\ell, \tau) d \ell=0, \quad \forall k \neq 0,
$$

with kernel $S$ given by

$$
S(k, \ell)=\frac{\Lambda(k, \ell)}{|k \ell(k+\ell)|^{1 / 2}}
$$


The definition of $S$ is extended by setting

$$
S(k, \ell)=0 \quad \text { if } k \ell=0 .
$$

The corresponding spatial form of (61) is

$$
\partial_{\tau} \psi+\partial_{\theta} a(\psi, \psi)=0
$$

where the bilinear form $a$ is defined by

$$
\widehat{a(\psi, \phi})(k, \tau)=\int_{-\infty}^{+\infty} S(k-\ell, \ell) \hat{\psi}(k-\ell, \tau) \hat{\phi}(\ell, \tau) d \ell .
$$

(63) has the form of a nonlocal Burgers equation, like (2.8) in [7], or (1.1) in [3].

We consider the initial value problem for the noncanonical equation (63), (64), supplemented by an initial condition

$$
\psi(\theta, 0)=\psi_{0}(\theta) .
$$

The well-posedness of (63)-(65) easily follows by adapting the proof of Hunter [7] (given for the periodic setting) to our case.

Theorem 3. For any $\psi_{0} \in H^{s}(\mathbb{R}), s>2$, the initial value problem (63)-(65) has a unique local solution

$$
\psi \in C\left(I ; H^{s}(\mathbb{R})\right) \cap C^{1}\left(I ; H^{s-1}(\mathbb{R})\right)
$$

defined on the time interval $I=\left(-\tau_{*}, \tau_{*}\right)$, where

$$
\tau_{*}=\frac{1}{K_{s}\left\|\psi_{0}\right\|_{L^{2}(\mathbb{R})}^{1-2 / s}\left\|\psi_{0}\right\|_{H^{s}(\mathbb{R})}^{2 / s}}
$$

for a suitable constant $K_{s}$.

For a complete proof of Theorem 3 see [11]. The well-posedness result of Theorem 3 may be easily recast as a similar result for (58), (59).

Sketch of proof. The proof is based on the a priori estimate

$$
\left|\frac{d}{d \tau}\|\psi\|_{H^{s}(\mathbb{R})}\right| \leq C C_{s}\left\|\psi_{0}\right\|_{L^{2}(\mathbb{R})}^{1-2 / s}\|\psi\|_{H^{s}(\mathbb{R})}^{1+2 / s},
$$

deduced from the properties (60) of the kernel. Using Gronwall's inequality, we deduce from (67) the bound

$$
\|\psi(\cdot, \tau)\|_{H^{s}(\mathbb{R})} \leq\left\|\psi_{0}\right\|_{H^{s}(\mathbb{R})}\left(1-\frac{2 C C_{s}}{s}\left\|\psi_{0}\right\|_{L^{2}(\mathbb{R})}^{1-2 / s}\left\|\psi_{0}\right\|_{H^{s}(\mathbb{R})}^{2 / s}|\tau|\right)^{-s / 2},
$$

for $|\tau|<\tau_{*}$ where $\tau_{*}$ is given by (66).

The rest of the proof follows from a standard Galerkin approximation. Given any function $f \in H^{s}(\mathbb{R})$ with Fourier transform $\hat{f}$ we define the finite dimensional orthogonal projection

$$
f^{N}(\theta)=P_{N} f(\theta)=\int_{|k| \leq N} \widehat{f}(k) e^{i k \theta} d k .
$$

We consider the Galerkin approximation $\psi^{N}=P_{N} \psi$, defined as the solution of the approximate system of ODEs

$$
\left\{\begin{array}{l}
\partial_{\tau} \psi^{N}+P_{N} \partial_{\theta} a\left(\psi^{N}, \psi^{N}\right)=0, \\
\psi^{N}(\theta, 0)=P_{N} \psi_{0}(\theta) .
\end{array}\right.
$$

The approximate solutions $\varphi^{N}$ satisfy the a priori estimate (68), uniformly in $N$. By standard arguments we can extract a subsequence $\left\{\varphi^{N}\right\}$ and pass to the limit in (69) to obtain a solution of (63). The uniqueness of the solution follows by a standard argument. See [11] for details.

In [11] we also obtain the following blow-up criterion. 
Lemma 4. Under the assumptions of Theorem 3, if $\psi \in C\left(0, T ; H^{s}(\mathbb{R})\right)$ with $0<T<+\infty$ is a solution of (63) such that

$$
\int_{0}^{T}\|\psi(\cdot, \tau)\|_{s^{\prime}}^{2 / s^{\prime}} d \tau<+\infty
$$

for some $s^{\prime}>2$, then $\psi$ is continuable to a solution $\psi \in C\left(0, T^{\prime} ; H^{s}(\mathbb{R})\right)$ with $T^{\prime}>T$.

Proof. See [11].

The resolution of (58), (59) via the introduction of the noncanonical variable $\psi(\theta, \tau)$, stated in Theorem 3 , is the chief step. When this is achieved it is not difficult to obtain the regularity of the other first order terms (29), (30) of the asymptotic expansion (9), as in the following lemma.

Lemma 5. For any $\psi_{0} \in H^{s}(\mathbb{R})$, with integer $s>2$, let

$$
\psi \in C\left(I ; H^{s}(\mathbb{R})\right) \cap C^{1}\left(I ; H^{s-1}(\mathbb{R})\right)
$$

be the solution of (63)-(65), given by Theorem 3. Let $\varphi^{(1)}$ be defined by $\psi(\theta, \tau)=\left|\partial_{\theta}\right|^{1 / 2} \varphi^{(1)}(\theta, \tau)$, and $\hat{U}^{(1)}, \hat{V}^{(1)}$ be defined by (29), (30). Then

$$
U^{(1)} \in C\left(I ; H^{s}\left(\mathbb{R}_{+}^{2}\right)\right) \cap C^{1}\left(I ; H^{s-1}\left(\mathbb{R}_{+}^{2}\right)\right), \quad V^{(1)} \in C\left(I ; H^{s}\left(\mathbb{R}_{-}^{2}\right)\right) \cap C^{1}\left(I ; H^{s-1}\left(\mathbb{R}_{-}^{2}\right)\right),
$$

where $\mathbb{R}_{ \pm}^{2}=\{(\theta, \eta) \mid \theta \in \mathbb{R}, \pm \eta>0\}$.

Proof. For the proof it is convenient to introduce the homogeneous space $\dot{H}^{m}(\mathbb{R})$ (w.r.to the independent variable $\theta$ ),

$$
\dot{H}^{m}(\mathbb{R})=\left\{u(\theta): \mathbb{R} \rightarrow \mathbb{R}: \int_{-\infty}^{+\infty}|k|^{2 m}|\hat{u}(k)|^{2} d k<+\infty\right\}
$$

normed by

$$
\|u\|_{\dot{H}^{m}(\mathbb{R})}=\left(\int_{-\infty}^{+\infty}|k|^{2 m}|\hat{u}(k)|^{2} d k\right)^{1 / 2} .
$$

Let us consider integers $m, p \geq 0$ such that $m+p \leq s$. For every $\tau \in I$ we have

$$
\left\|\partial_{\eta}^{p} U^{(1)}(\cdot, \tau)\right\|_{L^{2}\left(\mathbb{R}_{\eta}^{+} ; \dot{H}^{m}\left(\mathbb{R}_{\theta}\right)\right)}^{2}=\left\||k|^{m} \partial_{\eta}^{p} \hat{U}^{(1)}(\cdot, \tau)\right\|_{L^{2}\left(\mathbb{R}_{+}^{2}\right)}^{2},
$$

recalling that our Fourier transform is taken w.r.to $\theta$. Substituting the definition (29) gives

$$
\begin{aligned}
\left\||k|^{m} \partial_{\eta}^{p} \hat{U}^{(1)}(\cdot, \tau)\right\|_{L^{2}\left(\mathbb{R}_{+}^{2}\right)}^{2} & =\int_{0}^{\infty} \int_{\mathbb{R}}|k|^{2 m+2}\left|\hat{\varphi}^{(1)}(k, \tau)\right|^{2}\left|\partial_{\eta}^{p} e^{-|k| \eta}\right|^{2}|R|^{2} d k d \eta \\
= & \frac{1}{2}|R|^{2} \int_{0}^{\infty} \int_{\mathbb{R}}|k|^{2 m+2 p+1}\left|\hat{\varphi}^{(1)}(k, \tau)\right|^{2} 2|k| e^{-2|k| \eta} d k d \eta \\
& =\left.\left.\frac{1}{2}|R|^{2} \int_{\mathbb{R}}|k|^{2 m+2 p}|| k\right|^{1 / 2} \hat{\varphi}^{(1)}(k, \tau)\right|^{2} d k=\frac{1}{2}|R|^{2}\|\psi(\cdot, \tau)\|_{\dot{H}^{m+p}(\mathbb{R})}^{2}
\end{aligned}
$$

Adding over $m, p \geq 0$ such that $m+p \leq s$ in (71), (72) gives $U^{(1)} \in L^{\infty}\left(I ; H^{s}\left(\mathbb{R}_{+}^{2}\right)\right)$. The same calculation for a difference at times $\tau_{1}, \tau_{2}$ gives

$$
\left\|\partial_{\eta}^{p} U^{(1)}\left(\cdot, \tau_{1}\right)-\partial_{\eta}^{p} U^{(1)}\left(\cdot, \tau_{2}\right)\right\|_{L^{2}\left(\mathbb{R}_{\eta}^{+} ; \dot{H}^{m}\left(\mathbb{R}_{\theta}\right)\right)} \leq C\left\|\psi\left(\cdot, \tau_{1}\right)-\psi\left(\cdot, \tau_{2}\right)\right\|_{\dot{H}^{m+p}(\mathbb{R})},
$$

and adding again over $m+p \leq s$ gives the time continuity of $U^{(1)}$ in $H^{s}\left(\mathbb{R}_{+}^{2}\right)$, by the time continuity of $\psi$ in $H^{s}(\mathbb{R})$. Thus we have obtained $U^{(1)} \in C\left(I ; H^{s}\left(\mathbb{R}_{+}^{2}\right)\right)$. The proof of $U^{(1)} \in C^{1}\left(I ; H^{s-1}\left(\mathbb{R}_{+}^{2}\right)\right)$ is similar, thanks to the time continuity of $\psi_{\tau}$ in $H^{s-1}(\mathbb{R})$. By the same arguments we prove the regularity of $V^{(1)}$. 


\section{REFERENCES}

[1] G. Alì and J. K. Hunter. Nonlinear surface waves on a tangential discontinuity in magnetohydrodynamics. Quart. Appl. Math., 61(3):451-474, 2003.

[2] G. Alì, J. K. Hunter, and D. F. Parker. Hamiltonian equations for scale-invariant waves. Stud. Appl. Math., 108(3):305$321,2002$.

[3] S. Benzoni-Gavage. Local well-posedness of nonlocal Burgers equations. Differential Integral Equations, 22(3-4):303$320,2009$.

[4] J.P. Goedbloed, S. Poedts. Principles of magnetohydrodynamics with applications to laboratory and astrophysical plasmas. Cambridge University Press, Cambridge, 2004.

[5] M.F. Hamilton, Yu. A. Il'insky, and E. A. Zabolotskaya. Evolution equations for nonlinear Rayleigh waves. J. Acoust. Soc. Am., 97:891-897, 1995.

[6] J. K. Hunter. Nonlinear surface waves. In Current progress in hyberbolic systems: Riemann problems and computations (Brunswick, ME, 1988), pages 185-202. Amer. Math. Soc., 1989.

[7] J. K. Hunter. Short-time existence for scale-invariant Hamiltonian waves. J. Hyperbolic Differ. Equ., 3(2):247-267, 2006.

[8] J. K. Hunter. Nonlinear hyperbolic surface waves. In Nonlinear conservation laws and applications, volume 153 of IMA Vol. Math. Appl., pages 303-314. Springer, New York, 2011.

[9] N. Mandrik, Y. Trakhinin. Influence of vacuum electric field on the stability of a plasma-vacuum interface. Commun. Math. Sci., 12(6):1065-1100, 2014.

[10] A. Marcou. Rigorous weakly nonlinear geometric optics for surface waves. Asymptot. Anal., 69(3-4):125-174, 2010.

[11] P. Secchi. Nonlinear surface waves on the plasma-vacuum interface. Quart. Appl. Math., 2015, to appear.

Dicatam, Mathematical Division, University of Brescia, Via Branze 43, 25123 Brescia, Italy

E-mail address: paolo.secchi@unibs.it 